\title{
Termination Analysis by Learning Terminating Programs
}

\author{
Matthias Heizmann, Jochen Hoenicke, and Andreas Podelski \\ University of Freiburg, Germany
}

\begin{abstract}
We present a novel approach to termination analysis. In a first step, the analysis uses a program as a black-box which exhibits only a finite set of sample traces. Each sample trace is infinite but can be represented by a finite lasso. The analysis can "learn" a program from a termination proof for the lasso, a program that is terminating by construction. In a second step, the analysis checks that the set of sample traces is representative in a sense that we can make formal. An experimental evaluation indicates that the approach is a potentially useful addition to the portfolio of existing approaches to termination analysis.
\end{abstract}

\section{Introduction}

Termination analysis is an active research topic, and a wide range of methods and tools exist $1214 / 2327 / 293639$. Each method provides its own twist to address the same issue: in the presence of loops with branching or nesting, the termination argument has to account for all possible interleavings between the different paths through the loop.

If the program is lasso-shaped (a stem followed by a single loop without branching), the control flow is trivial; there is only one path. Consequently, the termination argument can be very simple. Many procedures are specialized to lasso-shaped programs and derive a simple termination argument rather efficiently 4 15:7 11 24:31 33. The relevance of lasso-shaped programs stems from their use as the representation of an infinite trace through the control flow graph of a program with arbitrary nesting.

We present a new method that analyzes termination of a general program $\mathcal{P}$ but has to find termination arguments only for lasso-shaped programs. In our method we see the program $\mathcal{P}$ as a blackbox from which we can obtain sample traces. We transform a sample trace $\pi_{i}$ into a lasso-shaped program and use existing methods to compute a termination argument for this lasso-shaped program. Afterwards we construct a "larger" program $\mathcal{P}_{i}$ (which may have branching and nested loops) for which the same termination argument is applicable. We call this construction learning, because we learned the terminating program $\mathcal{P}_{i}$ from a sample trace $\pi_{i}$. Our algorithm continues iteratively until we learned a set of

\footnotetext{
* This work is supported by the German Research Council (DFG) as part of the Transregional Collaborative Research Center "Automatic Verification and Analysis of Complex Systems" (SFB/TR14 AVACS).
}

A. Biere and R. Bloem (Eds.): CAV 2014, LNCS 8559, pp. 797-813, 2014.

(C) Springer International Publishing Switzerland 2014 
programs $\mathcal{P}_{1}, \ldots, \mathcal{P}_{n}$ that forms a decomposition of the original program $\mathcal{P}$. This decomposition can be seen as a program of the form choose $\left(\mathcal{P}_{1}, \ldots, \mathcal{P}_{n}\right)$, i.e., a nondeterministic choice of programs $\mathcal{P}_{1}, \ldots, \mathcal{P}_{n}$, that is semantically equivalent to the original program $\mathcal{P}$.

Our technical contribution is this method, which does not only extend the existing portfolio of termination analyses but also provides a new functionality: the decomposition of a program $\mathcal{P}$ into modules $\mathcal{P}_{1}, \ldots, \mathcal{P}_{n}$. This decomposition is not guided by the syntax of the program, this decomposition exploits a novel notion of modularity where a module is defined by a certain termination argument. This novel notion of modularity is the conceptual contribution of our paper.
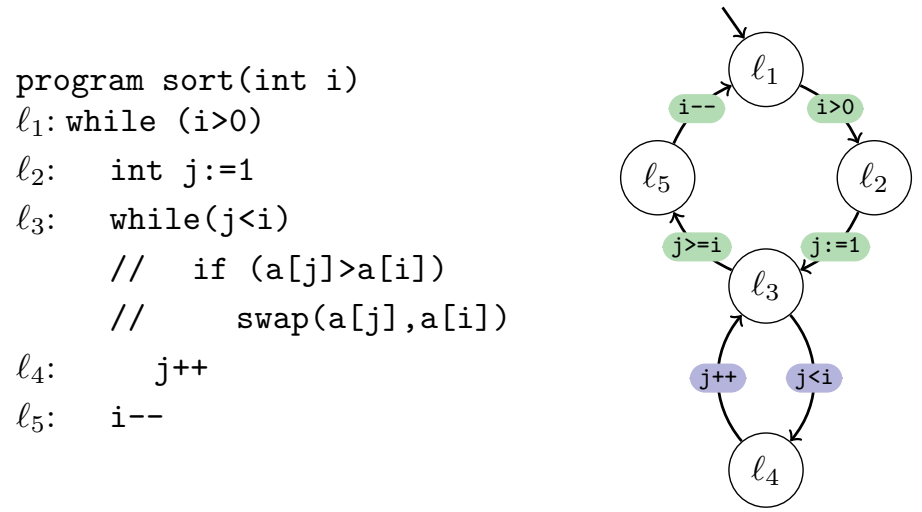

Let us explain our algorithm informally using the program $\mathcal{P}^{\text {sort }}$ depicted above which is an implementation of bubblesort. Termination of $\mathcal{P}^{\text {sort }}$ can be shown, e.g., by using the quadratic ranking function $f(i, j)=i^{2}-j$, or the lexicographic ranking function $f(i, j)=(i, i-j)$. Intuitively, neither of the two ranking functions is a simple termination argument.

Now, let us pick some $\omega$-trace from $\mathcal{P}^{\text {sort }}$. We take the trace that first enters the outer while loop and then takes the inner while loop infinitely often. We denote this trace using the $\omega$-regular expression OUTER.InNER ${ }^{\omega}$. We see that this trace is terminating. Its termination can be shown using the linear ranking function $f(i, j)=i-j$. Moreover, we see that this ranking function is not only applicable to this trace, this ranking function is applicable to all traces that eventually always take the inner loop.

$$
(\text { INNER }+ \text { OUTER })^{*} \cdot \text { INNER }^{\omega}
$$

Now, let us pick another $\omega$-trace from $\mathcal{P}^{\text {sort }}$. This time we take the trace that always takes the outer while loop. We see that this trace is terminating. Its termination can be shown using the linear ranking function $f(i, j)=i$. Moreover, we see that this ranking function is not only applicable to this trace, this ranking function is applicable to all traces that take the outer while loop infinitely often.

$$
(\text { INNER*.OUTER })^{\omega}
$$


Finally, we consider the set of all $\omega$-trace of the program $\mathcal{P}^{\text {sort }}$

$$
(\text { OUTER + INNER })^{\omega}
$$

check that each trace has the form (1) or has the form (2), and conclude that $\mathcal{P}^{\text {sort }}$ is terminating.

If we are to automate the reasoning from the example above, a number of questions arise.

(A) How does one effectively represent a set of traces that share a common reason for termination, like the sets (1) and (2) above? The answer is given in Section 2 where we define a module, which is a program whose traces adhere to a certain fairness constraint.

(B) What is a termination argument whose applicability to a whole set of traces can be checked effectively? The answer is given in Section 3 where we present a Floyd-Hoare style annotation for termination proofs.

(C) How can we learn a set of terminating traces (represented as a program with a fairness constraint) from a single terminating sample trace? The answer is given in Section 4 where we construct a terminating module from a given termination proof.

(D) How can we check that a set of modules $\mathcal{P}_{1}, \ldots, \mathcal{P}_{n}$ covers the behavior of the original program $\mathcal{P}$ and can we always decompose $\mathcal{P}$ into a set of modules $\mathcal{P}_{1}, \ldots, \mathcal{P}_{n}$ ? One facet of the question is the theoretical completeness, which is answered in Section 5. The other facet is the practical feasibility, which is analyzed via an experimental evaluation in Section 6 .

\section{Fair Module}

Preliminaries. The key concept in our formal exposition is the notion of an $\omega$-trace, which is an infinite sequence of program statements $\pi=s t_{1} s_{2} \ldots$ We assume that the statements are taken from a given finite set of program statements $\Sigma$. If we consider $\Sigma$ as an alphabet and each statement as a letter, then an $\omega$-trace is an infinite word over this alphabet. In order to stress the usage of statements as letters of an alphabet, we sometimes frame each statement/letter. For example, we can write the alphabet of our running example $\mathcal{P}^{\text {sort }}$ as $\Sigma_{\text {sort }}=$ $\{i>0, j:=1, j<i, j++, j>=i, i--\}$ and $\pi=j^{j} i j:=1 .\left(j:=1 j^{++} j:=1\right)^{\omega}$ is an $\omega$-trace.

The definition of an $\omega$-trace as an arbitrary (infinite) sequence means that the notion is independent of the programming language semantics, which we even have not introduced yet. We will do so now. A valuation $\nu$ is a function that maps the program variables $\vec{v}$ to values. We use the term valuation instead of state to stress that this is independent from the program counter (and independent from control flow). We call a set of valuations a predicate and use the letter $I$ to denote predicates. The letter $I$ is used reminiscent to invariant, because we will use predicates to represent invariants at locations. We assume that each statement st comes with a binary relation over the set of valuations (the set of its precondition/postcondition pairs). We say that the Hoare triple $\{I\} \operatorname{st}\left\{I^{\prime}\right\}$ is 
valid, if the binary relation for st holds between precondition $I$ and postcondition $I^{\prime}$. We use the interleaved sequences of valuations and statements $\nu_{0} \stackrel{s t_{1}}{\rightarrow} \stackrel{s t_{n}}{\rightarrow} \nu_{n}$ as a shorthand to denote that each pair of valuations $\left(\nu_{i}, \nu_{i+1}\right)$ is contained in the transition relation of the statement $s_{i+1}$.

An $\omega$-trace may not correspond to any possible execution for one out of two reasons. First, there may be a finite prefix that does not have any possible execution, like e.g., the prefix $x<0 \quad x:=1 \quad x<0$ of the $\omega$-trace $(x<0 \quad x:=1)^{\omega}$. Secondly, there may be no starting valuation $\nu_{0}$ for any infinite execution, although every finite prefix is executable which holds e.g., for the $\omega$-trace $\left(x>=0 x^{++}\right)^{\omega}$. In both cases we call such an $\omega$-trace terminating.

The notion of an $\omega$-trace is also independent of a program (a trace may not correspond to a path in the program's control flow graph). We introduce a program as a control flow graph whose edges are labeled with statements. Formally, a program is a graph $\mathcal{P}=\left\langle\right.$ Loc, $\left.\delta, \ell_{\text {init }}\right\rangle$ with a finite set Loc of nodes called locations, a set $\delta$ of edges labeled with statements, i.e., $\delta \subseteq \operatorname{Loc} \times \Sigma \times$ Loc and an initial node called the initial location $\ell_{\text {init }}$. We call the program $\mathcal{P}$ terminating if each of its $\omega$-traces is terminating.

Module: Program with Fairness Contraint. In our method we will decompose a program into modules such that each module represents traces that share a common reason for termination. We now formalize our notion of a module.

Definition 1 (module). A module is a program together with a fairness constraint given by a distinguished location $\ell_{\mathrm{fin}}$, i.e.,

$$
\mathcal{P}=\left\langle\text { Loc }, \delta, \ell_{\text {init }}, \ell_{\text {fin }}\right\rangle
$$

where the set of location can be partitioned into two disjoint sets, $\operatorname{Loc}_{U}$, and $\operatorname{Loc}_{V}$, such that

- the initial location is contained in $\operatorname{Loc}_{U}$,

- the final location is contained in $\operatorname{Loc}_{V}$, and

- no location in $\operatorname{Loc}_{V}$ has a successor in $\operatorname{Loc}_{U}$, i.e.,

$$
\left(\ell, s t, \ell^{\prime}\right) \in \delta \quad \text { implies } \quad \ell \in \operatorname{Loc}_{U} \quad \text { or } \quad \ell^{\prime} \in \operatorname{Loc}_{V}
$$

$A$ fair $\omega$-trace of a module $\mathcal{P}$ is an $\omega$-trace that labels a fair path in the graph of $\mathcal{P}$, which is a path that visits the distinguished location $\ell_{\mathrm{fin}}$ infinitely often. We call the module $\mathcal{P}$ terminating if each of its fair $\omega$-traces is terminating.

A non-fair $\omega$-trace of a terminating module (i.e., an $\omega$-trace that labels a path in its control flow graph without satisfying the fairness constraint) can be non-terminating.

For the reader who is familiar with the concept of Büchi automata, a module is reminiscent of a Büchi automaton with exactly one final state. A Büchi automaton of this form recognizes an $\omega$-regular language of the form $U . V^{\omega}$, where $U$ and $V$ are regular languages over the alphabet of statements $U, V \subseteq \Sigma^{*}$. 
Example 1. Let us consider again our running example $\mathcal{P}^{\text {sort }}$. The sets that we gave informally by the $\omega$-regular expressions (1) and (2) can be represented as modules. The module $\mathcal{P}_{1}^{\text {sort }}$ depicted on the left represents all traces that eventually only take the inner while loop. The
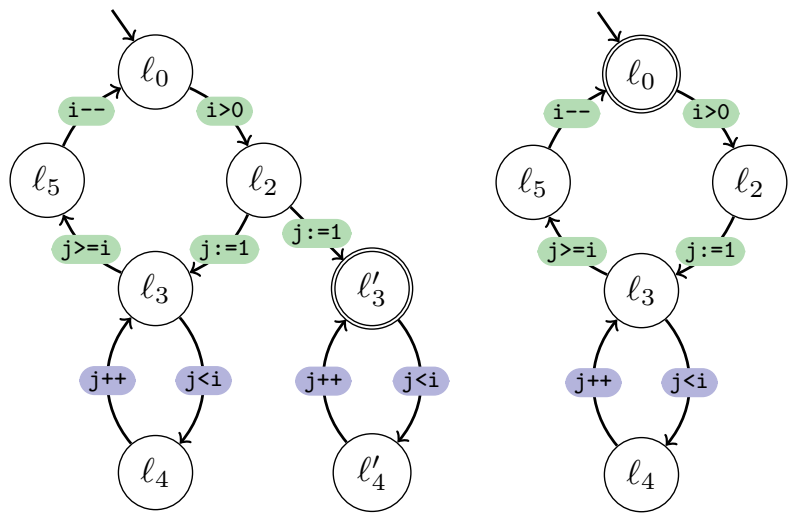
module $\mathcal{P}_{2}^{\text {sort }}$ depicted on the right represents all traces that take the outer while loop infinitely often.

In this example, the decomposition of the program into modules is defined by the nestings structure of while loops. In Section 5 we present an algorithm that finds a decomposition automatically but does not rely on any information about the structure of the while loops in the program.

\section{Certified Module}

In this section we present a termination argument for modules that consists of two parts: a ranking function and an annotation of the module's locations.

First, we extend the usual notion of a ranking function to our definition of a module. The crux in the following definition lies in the fact that we do not require that the value of the ranking function has to decrease after a fixed number of steps. We only require that the value of the ranking function has to decrease every time the final location $\ell_{\text {fin }}$ is visited. As a consequence our ranking function is a termination argument that is applicable to each fair $\omega$-trace, but does not have to take non-fair $\omega$-traces into account.

Definition 2 (ranking function for a module). Given a module $\mathcal{P}$, we call a function $f$ from valuations into a well-ordered set $(\mathbb{W}, \prec)$ a ranking function for $\mathcal{P}$ if for each finite path

$$
\ell_{0} \stackrel{s t_{1}}{\rightarrow} \cdots \stackrel{s t_{k}}{\rightarrow} \ell_{k} \stackrel{s t_{k+1}}{\rightarrow} \cdots \stackrel{s t_{n}}{\rightarrow} \ell_{n}
$$

that starts in the initial location (i.e., $\ell_{0}=\ell_{\text {init }}$ ) and visits the final location in the $k$-th step and in the $n$-th step (i.e., $\ell_{k}=\ell_{n}=\ell_{\text {fin }}$ ) and for each sequence of valuations $\nu_{0}, \ldots, \nu_{n}$ such that the pair $\left(\nu_{i}, \nu_{i+1}\right)$ is in the transition relation of the statement $s_{i}$, i.e.,

$$
\nu_{0} \stackrel{s t_{1}}{\rightarrow} \cdots \stackrel{s t_{k}}{\rightarrow} \nu_{k} \stackrel{s t_{k+1}}{\rightarrow} \cdots \stackrel{s t_{n}}{\rightarrow} \nu_{n}
$$

the value of the ranking function decreases whenever $\ell_{\mathrm{fin}}$ is visited, i.e.,

$$
f\left(\nu_{n}\right) \prec f\left(\nu_{k}\right) .
$$


In all the following examples we take $\mathbb{Z}$ as domain of the program variables. Our well-ordered set $\mathbb{W}$ will be $(\mathbb{Z} \cup\{\infty\}, \prec)$. The ordering $\prec$ is the natural order restricted to pairs where the second operand is greater than or equal to zero (i.e., $a \prec b$ if and only if $a<b \wedge b \geq 0$ ).

Example 2. The function $f: \operatorname{dom} \rightarrow \mathbb{Z} \cup\{\infty\}$ defined as $f(i, j)=i-j$ is a ranking function for the module $\mathcal{P}_{1}^{\text {sort }}$ depicted in Example 1.

Lemma 1. If the module $\mathcal{P}$ has a ranking function $f$, then each fair trace of the module is terminating 1

How can we check that a function is a ranking function for a module? We next introduce a novel kind of annotation, called rank certificate that serves as a proof for this task. Informally, a rank certificate is a Floyd-Hoare annotation that ensures that the value of the ranking function has decreased whenever the final location $\ell_{\text {fin }}$ was visited. Therefore, we introduce an auxiliary variable oldrnk that represents the value of the ranking function at the previous visit of $\ell_{\text {fin }}$. Initially, the auxiliary variable oldrnk has the value $\infty$ which is a value strictly greater than all other values from our well-ordered $\mathbb{W}$.

Definition 3 (certified module). Given a module $\mathcal{P}=\left\langle\right.$ Loc, $\left.\delta, \ell_{\text {init }},\left\{\ell_{\text {fin }}\right\}\right\rangle$ and a function $f$ from valuations into a well-ordered set $(\mathbb{W}, \prec)$, we call a mapping $\mathcal{I}$ from locations to predicates a rank certificate for the function $f$ and the module $\mathcal{P}$ if the following properties hold.

- The initial location $\ell_{\text {init }}$ is mapped to the predicate where the auxiliary variable oldrnk has the value $\infty$, i. e.,

$$
\mathcal{I}\left(\ell_{\text {init }}\right) \Leftrightarrow \text { oldrnk }=\infty \text {. }
$$

- The accepting state is mapped to a predicate in which the value of the ranking function $f$ over the program variables is smaller than the value of the variable oldrnk, i.e.,

$$
\mathcal{I}\left(\ell_{\text {fin }}\right) \Rightarrow(f(\vec{v}) \prec o l d r n k)
$$

- The outgoing edges of non-accepting locations correspond to valid Hoare triples, i.e.,

$$
\{\mathcal{I}(\ell)\} \text { st }\left\{\mathcal{I}\left(\ell^{\prime}\right)\right\} \text { is valid for }\left(\ell, \text { st }, \ell^{\prime}\right) \in \delta, \ell \neq \ell_{\text {fin }}
$$

and outgoing edges of the final location correspond to valid Hoare triples if we insert an additional assignment statement that assigns the value of the ranking function to the auxiliary variable oldrnk, i.e.,

$$
\{\mathcal{I}(\ell)\} \text { oldrnk: }=f(\vec{v}) ; \text { st }\left\{\mathcal{I}\left(\ell^{\prime}\right)\right\} \text { is valid for }\left(\ell_{\text {fin }}, s t, \ell^{\prime}\right) \in \delta
$$

We call the triple $(\mathcal{P}, f, \mathcal{I})$ a certified module.

${ }^{1}$ An extended version of this paper that also contains the correctness proofs is available online. 
Example 3. The figure on the right depicts a certified module $\left(\mathcal{P}_{1}^{\text {sort }}, f, \mathcal{I}\right)$ where $f$ is the ranking function $f(i, j)=i-j$ and $\mathcal{I}$ is the mapping of locations to predicates indicated by writing the predicate beneath the location.

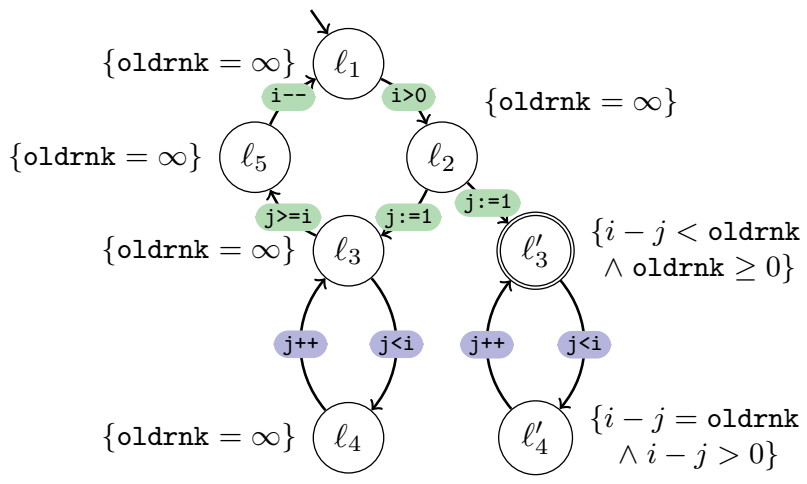

Theorem 1 (soundness). Each fair $\omega$-trace of a certified module $(\mathcal{P}, f, \mathcal{I})$ is terminating.

\section{Learning a Terminating Program}

In this section we present a method for the construction of a certified module $(\mathcal{P}, f, \mathcal{I})$. The crux of this method is that we do not construct a termination argument (a ranking function $f$ together with a rank certificate $\mathcal{I}$ ) for the resulting module $\mathcal{P}$. Instead, we construct vice versa the resulting module $\mathcal{P}$ as the largest module for which a given termination argument (a ranking function $f$ together with a rank certificate $\mathcal{I}$ ) is applicable. We obtain this termination proof from a single $\omega$-trace. We call this method learning, because we learn a terminating program (given as a certified module) from a single sample trace.

The input to our method is a terminating $\omega$-trace $s t_{1} \ldots s t_{k-1}\left(s t_{k} \ldots s t_{n}\right)^{\omega}$ that is ultimately periodic. We call an ultimately periodic trace a lasso. We call the prefix $s t_{1} \ldots s t_{k-1}$ the stem of the lasso and we call the periodic part $s t_{k} \ldots s t_{n}$ the loop of the lasso. For better legibility we use $u$ (resp. $v$ ) to denote the stem (resp. loop) of the lasso. We construct a certified module $(\mathcal{P}, f, \mathcal{I})$ in the following three steps.

\section{Step 1. Synthesize Ranking Function $f$}

First, we construct a module $\mathcal{P}_{u v^{\omega}}$ that has only one single $\omega$-trace, namely the lasso $u v^{\omega}$. We call $\mathcal{P}_{u v^{\omega}}$ the lasso module of $u v^{\omega}$ and construct $\mathcal{P}_{u v^{\omega}}=$ $\left\langle\right.$ Loc, $\left.\delta, \ell_{\text {init }},\left\{\ell_{\text {fin }}\right\}\right\rangle$ formally as the module that has one location for each statement (i.e., Loc $=\left\{\ell_{0}, \ldots, \ell_{n-1}\right\}$ ), where $\ell_{0}$ is the initial location, $\ell_{k}$ is the final location and the transition graph resembles the shape of a lasso, i.e., $\delta=\left\{\left(\ell_{i}, s_{i}, \ell_{i+1}\right) \mid i=1, \ldots n-2\right\} \cup\left\{\left(\ell_{n-1}, s_{n}, \ell_{k}\right)\right\}$.

The lasso module $\mathcal{P}_{u v}$ can be seen as a program that consists of a single while loop. This allows us to use existing methods [4/5/711/24/31|33] to synthesize a ranking function for $\mathcal{P}_{u v^{\omega}}$. 
Example 4. Given the $\omega$-trace $\mathrm{i}>0 \mathrm{j}:=1\left(\mathrm{j}^{\mathrm{i}} \mathrm{j}^{++}\right)^{\omega}$, we construct

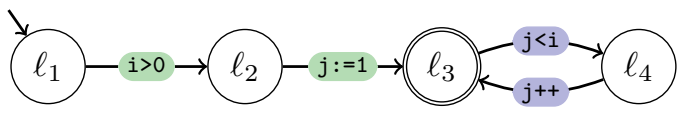
the lasso module $\mathcal{P}_{u v^{\omega}}$ depicted on the right and synthesize the ranking function $f(i, j)=i-j$ for this module.

\section{Step 2. Compute Rank Certificate $\mathcal{I}$}

Given the lasso module $\mathcal{P}_{u v^{w}}$ and the ranking function $f$, we now compute a rank certificate $\mathcal{I}$. Since $\mathcal{P}_{u v}$ has a "lasso shape" a mapping $\mathcal{I}$ from the locations of $\mathcal{P}_{u v^{\omega}}$ to predicates is a rank certificate if and only if the Hoare triples and the implication shown on the right are valid.

program rankDecrease()

$$
\begin{aligned}
& \text { oldrnk }:=\infty \\
& \ell_{1}: s t_{1} \\
& \ell_{k-1}: s_{k-1} \\
& \ell_{k} \text { : while (true) } \\
& \text { assert }(f(\vec{v})<\text { oldrnk }) \\
& \text { oldrnk }:=f(\overrightarrow{\mathrm{v}}) \\
& s t_{k} \\
& \ell_{k+1}: \quad s t_{k+1} \\
& \begin{array}{cc}
\vdots & \vdots \\
\ell_{n}: & s_{n}
\end{array}
\end{aligned}
$$

The predicates $\mathcal{I}\left(\ell_{i}\right)$ for which these implications are valid, can be obtained by proving partial correctness of the program rankDecrease $e_{u v f}$ depicted on the left. The program rankDecrease $e_{u v f}$ first assigns the value $\infty$ (which is strictly larger than any other element in the well-ordered set $\mathbb{W}$ ) to the variable oldrnk. Afterwards the statements $s t_{1} \ldots s t_{k-1}$ are executed and the program rankDecrease ${ }_{u v f}$ enters a nonterminating while loop. We use an assert statement to state the correctness specification of the program rankDecrease $u v f$. The program is correct if at the beginning of the while loop the inequality $f(\vec{v})<$ oldrnk holds. After this assert statement, the current value of the function $f$ is assigned to the variable oldrnk and then the statements $s t_{k} \ldots s t_{n}$ are executed.

A Floyd-Hoare annotation $\mathcal{I}\left(\ell_{1}\right), \ldots, \mathcal{I}\left(\ell_{n}\right)$ that shows partial correctness of the program rankDecrease $e_{u v f}$ is also a rank certificate for our ranking function $f$ and our lasso module $\mathcal{P}_{u v^{\omega}}$. This Floyd-Hoare annotation can be computed by static analysis [15].

Example 5. Continuing Example 4 we construct the program rankDecrease uvf $_{\text {for }}$ $\mathcal{P}_{u v w}$ and compute

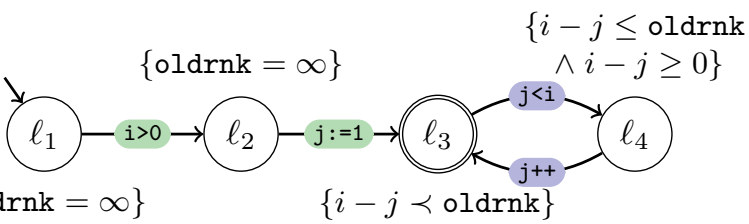
the rank certificate depicted in the figure on the right. The rank certificate $\mathcal{I}$ is represented by the predicates denoted beneath the locations. 
An Alternative Variant of Step 2. Some methods for the synthesis of a ranking function [724 also provide a supporting invariant. This is a predicate $I$ such that

- $I$ is invariant under executions of the loop $s t_{1} \ldots s t_{k-1}$,

- $I$ is an overapproximation of the reachable valuations after executing the stem $s t_{k} \ldots s t_{n}$,

- and each execution of the loop starting in a valuation contained $I$ decreases the ranking function $f$.

If we have a supporting invariant $I$ for the ranking function $f$, we do not have to construct and analyze the program rankDecrease $u v f$. Alternatively, we can set the predicate $\mathcal{I}\left(\ell_{k}\right)$ to

$$
I \wedge f(\vec{v})<\text { oldrnk } \wedge \text { oldrnk } \geq 0
$$

and obtain the remaining predicates $\mathcal{I}\left(\ell_{0}\right), \ldots, \mathcal{I}\left(\ell_{k-1}\right)$, and $\mathcal{I}\left(\ell_{k+1}\right), \ldots, \mathcal{I}\left(\ell_{n}\right)$ as strongest postconditions by using an interpolating theorem prover.

\section{Step 3. Construct Module $\mathcal{P}$}

We extend the lasso module $\mathcal{P}_{u v^{\omega}}$ to a module $\mathcal{P}$ that also has the ranking function $f$ and that also has the rank certificate $\mathcal{I}$. Therefore we modify $\mathcal{P}_{u v}$ according to the following two rules.

Modification Rule 1: Merge Locations. If the predicates mapped to the locations $\ell_{i}$ and $\ell_{j}$ coincide (i.e., $\mathcal{I}\left(\ell_{i}\right)=\mathcal{I}\left(\ell_{j}\right)$ ) then we may merge both locations.

Modification Rule 2: Add Transitions. Let st be some program statement and let $\ell_{i}$, and $\ell_{j}$ be locations. If $\ell_{i} \neq \ell_{j}$ and the Hoare triple $\left\{\ell_{i}\right\}$ st $\left\{\ell_{j}\right\}$ is valid, we may add the transition $\left(\ell_{i}, s t, \ell_{j}\right)$. If $\ell_{i}=\ell_{j}$ and the Hoare triple $\left\{\ell_{i}\right\}$ oldrnk: $=f(\vec{v}) ; s t\left\{\ell_{j}\right\}$ is valid, we may add the transition $\left(\ell_{i}, s t, \ell_{j}\right)$.

If we apply these modifications to a certified module we obtain again a certified module. Every strategy for applying these modfications gives rise to an algorithm that is an instance of our method.

Example 6. Continuing Example 4 we merge locations $\ell_{1}$ and $\ell_{2}$. Afterwards we add for each program statement that occurs in $\mathcal{P}^{\text {sort }}$ a selfloop at $\ell_{1}$ and

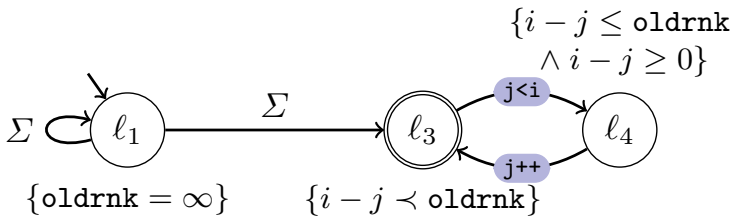

a transition between $\ell_{1}$ and $\ell_{3}$. We obtain the certified module $\mathcal{P}_{\text {ext }}$ depicted on the right. The set of fair $\omega$-traces of this module is given by the $\omega$-regular expression $\Sigma^{*} \cdot\left(\mathrm{j}_{<\mathrm{i}} \mathrm{j}^{++}\right)^{\omega}$. If we take the intersection of the program $\mathcal{P}^{\text {sort }}$ and the module $\mathcal{P}_{\text {ext }}$ we obtain the module $\mathcal{P}_{1}^{\text {sort }}$ from Example 1 . In our algorithm (Section [5), we do not need to construct modues such as $\mathcal{P}_{1}^{\text {sort }}$ explicitly (we only use their implicit representation through $\mathcal{P}_{\text {ext }}$ ). 


\section{Overall Algorithm}

Until now, we have formalized (and automated) one part of our method, which is to construct a terminating module from a given sample trace. We still need to formalize (and automate) how to check that a set of modules covers all behaviours of the program. We will say that the program $\mathcal{P}$ has a decomposition into the modules $\mathcal{P}_{1}, \ldots, \mathcal{P}_{n}$ if the set of $\omega$-traces of the program $\mathcal{P}$ is the union of the set of fair $\omega$-traces of the modules $\mathcal{P}_{1}, \ldots, \mathcal{P}_{n}$.

We can automate the check that indeed all cases are covered by reducing it to the inclusion between Büchi automata. Both a program and a module are special cases of Büchi automata (where the set of states is the set of program locations and the set of final states contains all program locations respectively the final location $\ell_{\text {fin }}$ only). By definition, the $\omega$-traces of the program $\mathcal{P}$ are exactly the infinite words accepted by the Büchi automaton $\mathcal{P}$ (and form the language $\mathcal{L}(\mathcal{P})$ recognized by $\mathcal{P}$ ), and the fair $\omega$-traces of the module $\mathcal{P}_{i}$ are exactly the infinite words accepted by the Büchi automaton $\mathcal{P}_{i}$ (and form the language $\mathcal{L}\left(\mathcal{P}_{i}\right)$ recognized by $\left.\mathcal{P}_{i}\right)$, for $i=1, \ldots, n$. The inclusion

$$
\mathcal{L}(\mathcal{P}) \subseteq \mathcal{L}\left(\mathcal{P}_{1}\right) \cup \cdots \cup \mathcal{L}\left(\mathcal{P}_{n}\right)
$$

can be checked by a model checker such as [26] or by a tool for manipulating Büchi automata such as 38 .

We will use Büchi automata also in order to prove that decomposing a program into certified modules is in principle a complete method for termination analysis.

Theorem 2 (completeness). If a program $\mathcal{P}$ is terminating then it can be decomposed into a finite set of certified modules, i.e., there are certified modules

$$
\left(\mathcal{P}_{1}, f_{1}, \mathcal{I}_{1}\right), \ldots,\left(\mathcal{P}_{n}, f_{n}, \mathcal{I}_{n}\right)
$$

such that the following equality holds.

$$
\mathcal{L}(\mathcal{P})=\mathcal{L}\left(\mathcal{P}_{1}\right) \cup \cdots \cup \mathcal{L}\left(\mathcal{P}_{n}\right)
$$

Overall Algorithm. Having reduced the check that a set of modules is a decomposition of a program, we are ready to present our algorithm for termination analysis, depicted below. The algorithm iteratively constructs certified modules $\left(\mathcal{P}_{i}, f_{i}, \mathcal{I}_{i}\right)$ until all $\omega$-traces of the program are known to be terminating or we encounter an $\omega$-trace for which we cannot find a termination argument.

At the beginning of each iteration (line 2) we check if there is an $\omega$-trace of the program $\mathcal{P}$ that is not already a fair $\omega$-trace of one of the modules $\mathcal{P}_{1}, \ldots, \mathcal{P}_{n-1}$ (for which termination has already been proven). As mentioned above, we reduce this check to language inclusion of Büchi automata. Therefore we know that whenever there exists a counterexample to language inclusion there exists also a lasso-shaped counterexample. We take such a lasso-shaped $\omega$-trace $u v^{\omega}$ and construct a program (called lasso module) whose only $\omega$-trace is $u v^{\omega}$ (see Step 1 in Section (4). Next, we analyze termination of the lasso module $\mathcal{P}_{u v^{\omega}}$. If 


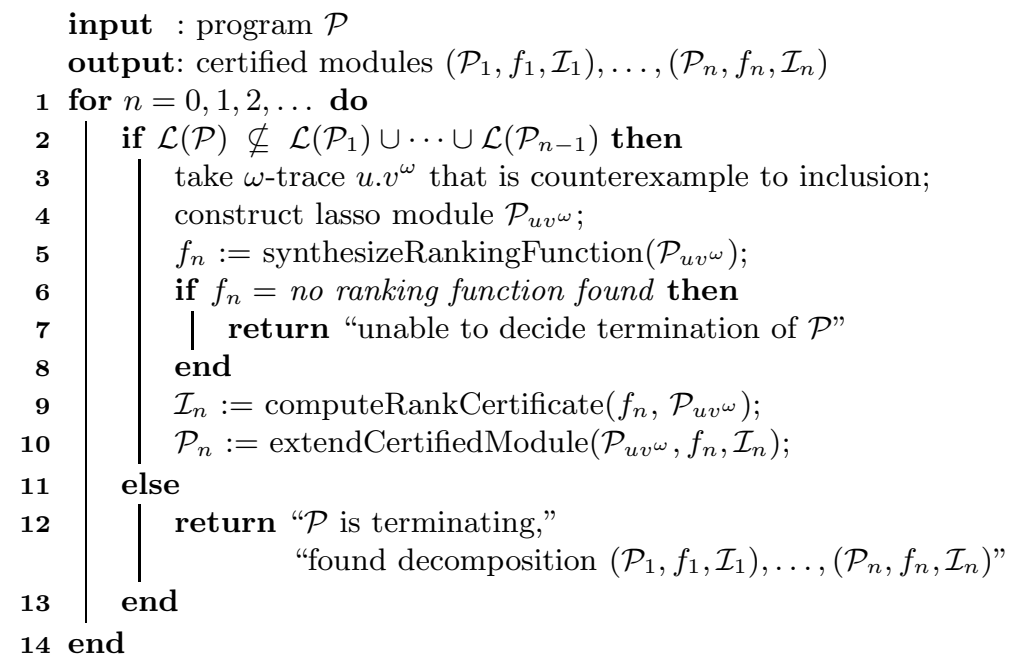

Algorithm 1. Decomposition of a $\operatorname{program} \mathcal{P}$ into certified modules

we cannot find a ranking function $f_{n}$ for $\mathcal{P}_{u v^{\omega}}$ our algorithm is unable to decide termination of $\mathcal{P}$ and returns. Otherwise we take a ranking function $f_{n}$ and construct a rank certificate $\mathcal{I}_{n}$ for $f_{n}$ and $\mathcal{P}_{u v^{\omega}}$ (see Step 2 in Section 4). Afterwards we use the rank certificate to construct the module $\mathcal{P}_{n}$. Termination of each fair $\omega$-trace of $\mathcal{P}_{n}$ can be shown using the ranking function $f_{n}$ and the rank certificate $\mathcal{I}_{n}$, i.e., $\left(\mathcal{P}_{n}, f_{n}, \mathcal{I}_{n}\right)$ is a certified module (see Step 3 in Section 4). If we were not able to find a counterexample to inclusion in line 2 , the program $\mathcal{P}$ is already decomposed into certified modules. We have proven termination and return the certified modules $\left(\mathcal{P}_{1}, f_{1}, \mathcal{I}_{1}\right), \ldots,\left(\mathcal{P}_{n}, f_{n}, \mathcal{I}_{n}\right)$.

Our approach lends itself to a variation of the above algorithm where one uses an exit condition different from the inclusion check in line 2 . In that case, the algorithm returns the modules $\mathcal{P}_{1}, \ldots, \mathcal{P}_{n-1}$ constructed so far and, in addition, a "remainder program" $\mathcal{P}_{\text {rem }}$ which is constructed via the language-theoretic difference of Büchi automata.

$$
\mathcal{P}_{\text {rem }}:=\mathcal{P} \backslash\left(\mathcal{P}_{1} \cup \cdots \cup \mathcal{P}_{n-1}\right)
$$

This is interesting in a variety of contexts, e.g., when we found an $\omega$-trace that is nonterminating, or when we found an $\omega$-trace whose termination analysis failed, or simply in case of a timeout. The remainder program can then be analyzed manually, or it can be used as a runtime monitor, etc.

\section{Evaluation}

It is unlikely that one approach outperforms all others on all kinds of programs, either in effectiveness (how many termination problems can be solved?) or in 
efficiency (... in what time?). In this paper, we have presented the base algorithm of a new approach to termination analysis. To explore optimizations and possibilities of integration with other approaches must remain a topic of future work.

The question is whether our approach is a potentially useful addition to the portfolio of existing approaches. Therefore, the goal of the present experimental evaluation must be restricted to showing that the approach has a practical potential in principle, regarding effectiveness and regarding efficiency. This is not obvious since there are at least two "mission-critical" questions, namely:

- Will the algorithm just learn one terminating program $\mathcal{P}_{1}, \mathcal{P}_{2}, \ldots$ after the other, going through an infinite (or just unrealistically high) number of sample traces $\pi_{1}, \pi_{2}, \ldots$ ?

- Will the check of inclusion between Büchi automata (which is notoriously difficult and still an object of ongoing work [837]) be a 'bad' bottleneck?

We put the evaluation into the context of a previous, very thorough evaluation 2 in [9] that contained 260 terminating programs. Out of the 260 programs, our tool can handle 236 programs. This, we believe, indicates the potential effectiveness of our approach. In comparison regarding effectiveness, CoOPERATINGT2, the "winner" of the evaluation in [9] (a highly optimized tool which integrates several approaches) can handle 14 programs that our tool cannot handle, but our tool can handle 5 programs that CoOpERATING-T2 cannot handle (namely a.10.c.t2.c, eric.t2.c, sas2.t2.c, spiral.t2.c and sumit.t2.c). This confirms our point that no single approach provides a "silver bullet" and that it is desirable to have a large portfolio of approaches.

We implemented the algorithm presented in Section 5 in the tool Ultimate BuchiAutomizer that analyzes termination of $\mathrm{C}$ programs. The input programs and the modules are represented by Büchi automata. In order to support (possibly recursive) functions, we use Büchi automata over nested words [1] (we do not introduce the formalism in order to avoid the notational overhead) and implemented an automata library for these automata. We do not check the inclusion

$$
\mathcal{L}(\mathcal{P}) \subseteq \mathcal{L}\left(\mathcal{P}_{1}\right) \cup \cdots \cup \mathcal{L}\left(\mathcal{P}_{n}\right)
$$

directly. Instead, we complement the modules and check the emptiness of their intersection with the program

$$
\mathcal{L}(\mathcal{P}) \cap \mathcal{L}\left(\overline{\mathcal{P}_{1}}\right) \cup \cdots \cup \mathcal{L}\left(\overline{\mathcal{P}_{n}}\right)
$$

which allow us to reuse intermediate results in further iterations. For complementing our Büchi automata we extended 41] the rank-based approach [18 to (Büchi) nested word automata. The sample $\omega$-traces whose termination we analyze are obtained as counterexamples of an emptiness check that is implemented in our automata library. This emptiness check is purely automata theoric, does not exploit any information about the program, but prefers short counterexamples. We use the tool LAssoRANKER 24/31] to synthesize ranking functions

\footnotetext{
2 http://verify.rwth-aachen.de/brockschmidt/Cooperating-T2/
} 
and supporting invariants for lassos. The Floyd-Hoare annotation is obtained via interpolation (alternative variant of Step 2 in Section 4). For interprocedural $\omega$-traces we resort to nested interpolants 25 . As interpolating theorem prover we use SMTInterpol [10. While constructing the modules, we apply Modification rule 1 (merge locations) always and we apply Modification rule 2 (add transitions) lazily in the following sense. Only if the automata library queries the existence of a transition in the module, we check whether this transition can be added by applying Modification rule 2. Our tool is available as a command line version for download as well as via a web interface at the following URL.

\section{http://ultimate.informatik.uni-freiburg.de/BuchiAutomizer/}

The following table shows the results for a subset of the benchmarks from 9 where our tool run on a computer with an Intel Core i5-3340M CPU with 2.70GHz. Our tool and as well as LASSORANKER the SMT solver, and the automata library are written in Java. The maximum heap size of the Java virtual machine was set to $4 \mathrm{~GB}(-\mathrm{Xmx} 4 \mathrm{G})$.

For each example we list the lines of code of this example, the overall runtime that our tool needed and the time that our tool spend for analyzing lassos, constructing modules, and checking language inclusion of Büchi automata. Furthermore, we list the number of certified modules that had a trivial ranking function (e.g., $f(x)=0$ ), the number of certified modules that had a non-trivial ranking function, and the number of states of the largest module that was constructed.

\begin{tabular}{|c|c|c|c|c|c|c|c|c|}
\hline filename & 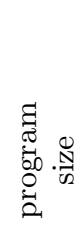 & 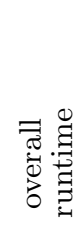 & 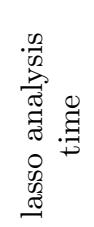 & 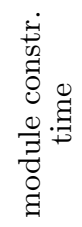 & 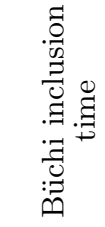 & 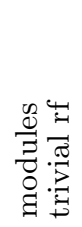 & 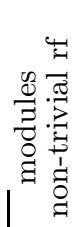 & 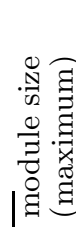 \\
\hline a.10.c.t2.c & 183 & $9 \mathrm{~s}$ & $2.8 \mathrm{~s}$ & $0.7 \mathrm{~s}$ & $2.1 \mathrm{~s}$ & 2 & 9 & 5 \\
\hline bf20.t2.c & 156 & $6 \mathrm{~s}$ & $0.7 \mathrm{~s}$ & $0.9 \mathrm{~s}$ & $1.9 \mathrm{~s}$ & 6 & 7 & \\
\hline bubbleSort.t2.c & 109 & $5 \mathrm{~s}$ & $0.7 \mathrm{~s}$ & $0.3 \mathrm{~s}$ & $1.2 \mathrm{~s}$ & 5 & 5 & \\
\hline consts1.t2.c & 40 & $2 \mathrm{~s}$ & $0.3 \mathrm{~s}$ & $0.1 \mathrm{~s}$ & $0.2 \mathrm{~s}$ & 2 & 1 & 5 \\
\hline edn.t2.c & 294 & $119 \mathrm{~s}$ & $18.8 \mathrm{~s}$ & $7.7 \mathrm{~s}$ & $89.0 \mathrm{~s}$ & 141 & 15 & 58 \\
\hline eric.t2.c & 53 & $10 \mathrm{~s}$ & $1.1 \mathrm{~s}$ & $1.7 \mathrm{~s}$ & $5.0 \mathrm{~s}$ & 4 & 6 & 14 \\
\hline firewire.t2.c & 178 & $28 \mathrm{~s}$ & $3.6 \mathrm{~s}$ & $1.3 \mathrm{~s}$ & $19.0 \mathrm{~s}$ & 12 & 7 & 8 \\
\hline mc91.t2.c & 47 & $12 \mathrm{~s}$ & $1.2 \mathrm{~s}$ & $0.6 \mathrm{~s}$ & $4.3 \mathrm{~s}$ & 4 & 10 & \\
\hline p-43-terminate.t2.c & 727 & $124 \mathrm{~s}$ & $2.1 \mathrm{~s}$ & $4.2 \mathrm{~s}$ & $110.6 \mathrm{~s}$ & 6 & 18 & \\
\hline reverse.t2.c & 1351 & $14 \mathrm{~s}$ & $3.1 \mathrm{~s}$ & $1.2 \mathrm{~s}$ & $2.9 \mathrm{~s}$ & 2 & 3 & 12 \\
\hline s3-work.t2.c & 3229 & $28 \mathrm{~s}$ & $2.1 \mathrm{~s}$ & $4.1 \mathrm{~s}$ & $11.5 \mathrm{~s}$ & 6 & 12 & 22 \\
\hline sas2.t2.c & 192 & $12 \mathrm{~s}$ & $1.3 \mathrm{~s}$ & $3.0 \mathrm{~s}$ & $5.5 \mathrm{~s}$ & 12 & 6 & 17 \\
\hline spiral.c & 65 & $38 \mathrm{~s}$ & $0.9 \mathrm{~s}$ & $1.3 \mathrm{~s}$ & $32.7 \mathrm{~s}$ & 8 & 12 & 14 \\
\hline sumit.t2.c & 83 & $4 \mathrm{~s}$ & $1.0 \mathrm{~s}$ & $0.2 \mathrm{~s}$ & $0.7 \mathrm{~s}$ & 4 & 2 & 4 \\
\hline traverse_twice.t2.c & 1428 & $12 \mathrm{~s}$ & $1.7 \mathrm{~s}$ & $1.4 \mathrm{~s}$ & $3.2 \mathrm{~s}$ & 2 & 4 & 18 \\
\hline ud.t2.c & 279 & $32 \mathrm{~s}$ & $2.1 \mathrm{~s}$ & $3.8 \mathrm{~s}$ & $22.1 \mathrm{~s}$ & 30 & 25 & 32 \\
\hline
\end{tabular}

More result: 3 of our tool can be found at the SV-COMP 2014 6] where our tool participated in the demonstration category on termination.

\footnotetext{
3 http://sv-comp.sosy-lab.org/2014/results/
} 
Discussion. A reader who is familiar with Büchi automata may wonder why it is feasible to complement Büchi automata of these sizes. The answer lies in the flexibility that our definition of a module allows. We tuned the construction of modules in a way that the "amount of nondeterminism" is kept low. However, it is still part of our future work to find a class of Büchi automata that can be easily complemented but does not hinder the module from accepting many traces.

\section{Related Work}

Our method is related to control flow refinement 22]. There, a multi-path loop is transformed into a semantically equivalent code fragment with simpler loops. For example, following the algebraic decomposition rule

$$
(a+b)^{*}=\left(b^{*} a b^{*}\right)^{+}+b^{*}
$$

the loop with the choice of two paths $a$ and $b$ is transformed into the nondeterministic choice of two loops, one where $a$ appears and one where it does not.

We extend control flow refinement by adding fairness constraints [40] and our reasoning is based on $\omega$-regular languages. In our running example (if we read $a$ as the outer and $b$ as the inner loop) we decomposed the $\omega$-regular expression describing the nested loops as follows

$$
(a+b)^{\omega}=(a+b)^{*} b^{\omega}+\left(b^{*} a\right)^{\omega} .
$$

We do not enforce the use of a fixed set of algebraic decomposition rules. Instead, we propose an algorithm that builds a decomposition on demand from simple termination arguments. Thus, we partition a set of traces only when it is necessary and, by construction, we produce only modules that are guaranteed to have a simple termination argument.

There are many other termination analyses, e. g., 314 161920 2139]. Most related are the termination analyses based on transition invariants and termination analyses based on size-change termination.

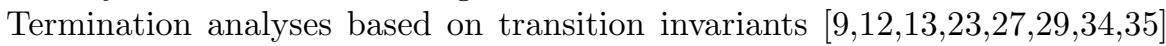
combine different, independently obtained ranking functions to a termination argument. Using transition invariants it is sufficient to cover finite repetitions of the loop. In our running example, one could cover the loop by

$$
(a+b)^{+}=b^{+}+\left(b^{*} a b^{*}\right)^{+}
$$

using the same simple ranking functions as our method for each case. Covering only finite traces is sound, as it can be shown that

$$
(a+b)^{\omega}=(a+b)^{*} b^{\omega}+(a+b)^{*}\left(b^{*} a b^{*}\right)^{\omega}
$$

using Ramsey's Theorem. In our approach, instead of having to introduce $(a+b)^{*}$, we can get a more precise characterization of the code before the infinite loop; 
also, we can base our case-distinction on which path was taken before the loop was reached. Furthermore, we get smaller expressions. Compare the expression

$$
(a+b)^{*}\left(b^{*} a b^{*}\right)^{\omega}
$$

with our expression $\left(b^{*} a\right)^{\omega}$. Although they describe exactly the same traces, our expression is simpler and therefore leads to a simpler termination proof. Redefining the loop entry point or unfolding the loops are intrinsic techniques in our approach (as opposed to add-on heuristics). If for the program $(a b)^{\omega}$, it is simpler to prove the correctness of the loop ( $b a b a)$, we use the fact that

$$
(a b)^{\omega}=a(b a b a)^{\omega} .
$$

The idea of size-change termination [4/7/28] is to track the value of (auxiliary) variables and show the absence of infinite executions by showing that one value would be decreased infinitely often in a well-ordered domain. The (auxiliary) variables can be seen as a predefined set of mutually independent termination arguments.

In contrast with the above approaches, a termination argument in our setting is a stand alone module (its validity is checked for the corresponding fair $\omega$ traces, independently from all other program traces). In contrast, a component of a lexicographic ranking function, a disjunct of a transition invariant, or a size-change variable makes sense only as part of a global termination argument (whose validity has to be checked for the global program).

Finally, we use "learning" as a metaphor rather than as a technical term, in contrast with the work in [30] which uses machine learning for termination analysis.

\section{Conclusion and Future Work}

We have presented a algorithm for termination analysis that transforms a program into a nondeterministic choice of programs. Our transformation is not guided by the syntactic structure of the program, but by its semantics. Instead of decomposing the program into modules and analyzing termination of the modules, we construct modules that we learned from sample traces and that are terminating by construction.

The general idea of such a transformation is the same as for trace refinement 32]: move disjunction over abstract values to the disjunction over sets of traces. The formalization of the shared idea and the exploration of its theoretical and practical consequences for program analyses is a topic of future work.

\section{References}

1. Alur, R., Madhusudan, P.: Adding nesting structure to words. J. ACM 56(3) (2009)

2. Apt, K.R., de Boer, F.S., Olderog, E.-R.: Verification of Sequential and Concurrent Programs, 3rd edn. Texts in Computer Science, p. 502. Springer (2009) ISBN 978$1-84882-744-8$ 
3. Babic, D., Hu, A.J., Rakamaric, Z., Cook, B.: Proving termination by divergence. In: SEFM, pp. 93-102 (2007)

4. Ben-Amram, A.M.: Size-change termination, monotonicity constraints and ranking functions. In: Bouajjani, A., Maler, O. (eds.) CAV 2009. LNCS, vol. 5643, pp. 109-123. Springer, Heidelberg (2009)

5. Ben-Amram, A.M., Genaim, S.: On the linear ranking problem for integer linearconstraint loops. In: POPL, pp. 51-62 (2013)

6. Beyer, D.: Status report on software verification - (competition summary sv-comp 2014). In: Ábrahám, E., Havelund, K. (eds.) TACAS 2014. LNCS, vol. 8413, pp. 373-388. Springer, Heidelberg (2014)

7. Bradley, A.R., Manna, Z., Sipma, H.B.: Linear ranking with reachability. In: Etessami, K., Rajamani, S.K. (eds.) CAV 2005. LNCS, vol. 3576, pp. 491-504. Springer, Heidelberg (2005)

8. Breuers, S., Löding, C., Olschewski, J.: Improved ramsey-based büchi complementation. In: Birkedal, L. (ed.) FOSSACS 2012. LNCS, vol. 7213, pp. 150-164. Springer, Heidelberg (2012)

9. Brockschmidt, M., Cook, B., Fuhs, C.: Better termination proving through cooperation. In: Sharygina, N., Veith, H. (eds.) CAV 2013. LNCS, vol. 8044, pp. 413-429. Springer, Heidelberg (2013)

10. Christ, J., Hoenicke, J., Nutz, A.: Smtinterpol: An interpolating smt solver. In: Donaldson, A., Parker, D. (eds.) SPIN 2012. LNCS, vol. 7385, pp. 248-254. Springer, Heidelberg (2012)

11. Cook, B., Kroening, D., Rümmer, P., Wintersteiger, C.M.: Ranking function synthesis for bit-vector relations. In: Esparza, J., Majumdar, R. (eds.) TACAS 2010. LNCS, vol. 6015, pp. 236-250. Springer, Heidelberg (2010)

12. Cook, B., Podelski, A., Rybalchenko, A.: Termination proofs for systems code. In: PLDI, pp. 415-426 (2006)

13. Cook, B., Podelski, A., Rybalchenko, A.: Proving program termination. Commun. ACM 54(5), 88-98 (2011)

14. Cook, B., See, A., Zuleger, F.: Ramsey vs. lexicographic termination proving. In: Piterman, N., Smolka, S.A. (eds.) TACAS 2013. LNCS, vol. 7795, pp. 47-61. Springer, Heidelberg (2013)

15. Cousot, P., Cousot, R.: Abstract interpretation: A unified lattice model for static analysis of programs by construction or approximation of fixpoints. In: POPL, pp. 238-252 (1977)

16. Cousot, P., Cousot, R.: An abstract interpretation framework for termination. In: POPL, pp. 245-258 (2012)

17. Fogarty, S., Vardi, M.Y.: Büchi complementation and size-change termination. Logical Methods in Computer Science 8(1) (2012)

18. Friedgut, E., Kupferman, O., Vardi, M.Y.: Büchi complementation made tighter. In: Wang, F. (ed.) ATVA 2004. LNCS, vol. 3299, pp. 64-78. Springer, Heidelberg (2004)

19. Ganty, P., Genaim, S.: Proving termination starting from the end. In: Sharygina, N., Veith, H. (eds.) CAV 2013. LNCS, vol. 8044, pp. 397-412. Springer, Heidelberg (2013)

20. Giesl, J., Thiemann, R., Schneider-Kamp, P., Falke, S.: Automated termination proofs with aProVE. In: van Oostrom, V. (ed.) RTA 2004. LNCS, vol. 3091, pp. 210-220. Springer, Heidelberg (2004)

21. Grebenshchikov, S., Lopes, N.P., Popeea, C., Rybalchenko, A.: Synthesizing software verifiers from proof rules. In: PLDI, pp. 405-416 (2012) 
22. Gulwani, S., Jain, S., Koskinen, E.: Control-flow refinement and progress invariants for bound analysis. In: PLDI, pp. 375-385 (2009)

23. Harris, W.R., Lal, A., Nori, A.V., Rajamani, S.K.: Alternation for termination. In: Cousot, R., Martel, M. (eds.) SAS 2010. LNCS, vol. 6337, pp. 304-319. Springer, Heidelberg (2010)

24. Heizmann, M., Hoenicke, J., Leike, J., Podelski, A.: Linear ranking for linear lasso programs. In: Van Hung, D., Ogawa, M. (eds.) ATVA 2013. LNCS, vol. 8172, pp. 365-380. Springer, Heidelberg (2013)

25. Heizmann, M., Hoenicke, J., Podelski, A.: Nested interpolants. In: POPL, pp. 471-482 (2010)

26. Holzmann, G.J.: The model checker spin. IEEE Trans. Software Eng. 23(5), 279-295 (1997)

27. Kroening, D., Sharygina, N., Tsitovich, A., Wintersteiger, C.M.: Termination analysis with compositional transition invariants. In: Touili, T., Cook, B., Jackson, P. (eds.) CAV 2010. LNCS, vol. 6174, pp. 89-103. Springer, Heidelberg (2010)

28. Lee, C.S., Jones, N.D., Ben-Amram, A.M.: The size-change principle for program termination. In: POPL, pp. 81-92 (2001)

29. Lee, W., Wang, B.-Y., Yi, K.: Termination analysis with algorithmic learning. In: Madhusudan, P., Seshia, S.A. (eds.) CAV 2012. LNCS, vol. 7358, pp. 88-104. Springer, Heidelberg (2012)

30. Lee, W., Wang, B.-Y., Yi, K.: Termination analysis with algorithmic learning. In: Madhusudan, P., Seshia, S.A. (eds.) CAV 2012. LNCS, vol. 7358, pp. 88-104. Springer, Heidelberg (2012)

31. Leike, J., Heizmann, M.: Ranking templates for linear loops. In: Ábrahám, E., Havelund, K. (eds.) TACAS 2014. LNCS, vol. 8413, pp. 172-186. Springer, Heidelberg (2014)

32. Mauborgne, L., Rival, X.: Trace partitioning in abstract interpretation based static analyzers. In: Sagiv, M. (ed.) ESOP 2005. LNCS, vol. 3444, pp. 5-20. Springer, Heidelberg (2005)

33. Podelski, A., Rybalchenko, A.: A complete method for the synthesis of linear ranking functions. In: Steffen, B., Levi, G. (eds.) VMCAI 2004. LNCS, vol. 2937, pp. 239-251. Springer, Heidelberg (2004)

34. Podelski, A., Rybalchenko, A.: Transition invariants. In: LICS, pp. 32-41 (2004)

35. Podelski, A., Rybalchenko, A.: Transition predicate abstraction and fair termination. In: POPL, pp. 132-144 (2005)

36. Popeea, C., Rybalchenko, A.: Compositional termination proofs for multi-threaded programs. In: Flanagan, C., König, B. (eds.) TACAS 2012. LNCS, vol. 7214, pp. 237-251. Springer, Heidelberg (2012)

37. Tsai, M.-H., Fogarty, S., Vardi, M.Y., Tsay, Y.-K.: State of Büchi complementation. In: Domaratzki, M., Salomaa, K. (eds.) CIAA 2010. LNCS, vol. 6482, pp. 261-271. Springer, Heidelberg (2011)

38. Tsai, M.-H., Tsay, Y.-K., Hwang, Y.-S.: Goal for games, omega-automata, and logics. In: Sharygina, N., Veith, H. (eds.) CAV 2013. LNCS, vol. 8044, pp. 883889. Springer, Heidelberg (2013)

39. Urban, C., Miné, A.: An abstract domain to infer ordinal-valued ranking functions. In: Shao, Z. (ed.) ESOP 2014. LNCS, vol. 8410, pp. 412-431. Springer, Heidelberg (2014)

40. Vardi, M.Y.: Verification of concurrent programs: The automata-theoretic framework. Ann. Pure Appl. Logic 51(1-2), 79-98 (1991)

41. $\mathrm{Wu}, \mathrm{X} .:$ Three operations on Büchi nested word automata for program verification. Master's thesis. University of Freiburg, Germany (2011) 